\title{
Notas sobre igualitarismo \\ y materialismo histórico ${ }^{1}$
}

Pablo Gres $C^{2}$

\section{Introducción}

En el presente trabajo, examinaremos el contenido filosófico-político que el debate igualitarista ha otorgado a la igualdad, con el objeto de poner a discutir a esta tradición con el materialismo histórico.

En un primer momento, nos interesa dar razones de peso para mostrar la urgencia de pensar la igualdad. En ese sentido, nos ocupará criticar la desigualdad actual.

En segundo lugar, nos detendremos a examinar los aportes del liberalismo igualitario y de la tradición en la que deviene, el igualitarismo. Revisaremos sus principales aportes y premisas filosóficas, que apuntan a dotar de contenido cierto a la idea de igualdad. Nos sumergiremos en su debate teórico, con el objeto de reconocer sus principales líneas argumentativas y los límites de las propuestas.

En la sección siguiente, atendiendo a los límites de la tradición igualitaria, nos ocuparemos de evidenciar los problemas y las insuficiencias teóricas de las que esta tradición adolece. Criticaremos al igualitarismo desde el materialismo histórico. No por mero capricho o voluntarismo político, sino más bien, porque el marxismo ofrece herramientas teorético-críticas potentes que permiten evidenciar que la desigualdad no es un

\footnotetext{
Este trabajo es una reformulación y ampliación de un artículo publicado en la revista Derecho y Humanidades de la U. de Chile, titulado Notas sobre Justicia Social y Materialismo Histórico. Cfr: Gres C., Pablo. 2013. Notas sobre Justicia Social y Materialismo Histórico, en: Revista Derecho y Humanidades, N 22, Santiago

2 Abogado, Universidad de Chile. Estudiante de Doctorado en la Facultad de Derecho, Universidad de Buenos Aires, Argentina
} 
mero síntoma, ni un problema contingente, sino que es una característica estructural de la formación social capitalista.

Sostenemos que el igualitarismo y el materialismo histórico son dos tradiciones que perfectamente pueden dialogar, básicamente porque miran con sospecha las relaciones sociales que conforman el modo de producción capitalista y la desigualdad que estas generan. En tal sentido, en la siguiente sección, posicionaremos a Marx en el debate igualitarista. Postularemos que existen insumos suficientes como para formular, desde la obra de Marx, principios igualitarios o de justicia social. Esos principios, dialogarán con la propuesta rawlsiana.

Finalmente, ofreceremos algunas reflexiones finales, encaminadas a pensar la igualdad más allá de las fronteras de aquella formación social que necesita la desigualdad, el capitalismo.

\section{Desigualdad y sirena de alarma}

A mediados del siglo XX, gracias a los aportes del positivismo jurídico, que encarnado en autores como Kelsen, sostuvo que "la justicia es una idea irracional" (Kelsen, 1957, p. 21); o los de la teoría económico política neoliberal y sus políticas que privilegiaron crecimiento económico en desmedro de la igualdad, se instauró un discurso en el que la justicia social no gozó de mucho respaldo académico. Por el contrario, se concebía a la justicia social como una abstracción filosófica o un atavismo cultural.

Como contraparte, casi al mismo tiempo, desde el liberalismo, surgieron algunas voces disidentes que veían en la justicia, particularmente en la igualdad, una cuestión central.

Hoy, hay razones de peso para sostener que la desigualdad no es un mal necesario. El problema de la desigualdad no es, en absoluto, un problema baladí, sobre todo teniendo en cuenta que al día de hoy, afecta estructuralmente a la sociedad.

Respecto de la abismante desigualdad global, "el filósofo alemán Thomas Pogge reunió evidencia estadística que provoca 
náuseas: en 1998, de un total de 5.820 millones de seres humanos, 1.214 millones poseían un ingreso de menos de un dólar norteamericano por día, y 2.800 millones vivían con menos de dos dólares por día" (Callinicos, 2006, p. 263). Más aún, "18 millones de personas mueren prematuramente cada año debido a causas vinculadas con la pobreza" (Mollard, 2012).

La pobreza masiva aumenta según pasan los años. La proporción del ingreso de la quinta parte más rica de la población mundial respecto de la quinta parte más pobre ha crecido de “30:1 en 1960 a 60:1 en 1990, y 74:1 en 1997" (UNDP, 1999). Hoy, según un informe de la OXFAM (OXFAM, 2016), casi la mitad de la riqueza mundial está en manos del $1 \%$ más rico y como contrapartida, "870 millones de personas no tienen lo suficiente para comer"(FAO, 2012). El mismo informe de la OXFAM, sostiene que el $1 \%$ de la población más rica acumula una fortuna equivalente a 65 veces la riqueza del $50 \%$ más pobre.

La desigualdad, claramente es un problema de nuestro tiempo, pero, a su vez, la igualdad es de uno u otro modo, un valor y una promesa esencialmente moderna. Ya en 1789, la revolución francesa prometía a sus ciudadanos: Libertad, Igualdad y Fraternidad. Más de 200 años después es totalmente lícito preguntarnos qué fue de aquella promesa. El asunto, hoy, ya no es sólo preocupante en términos estadísticos. "Si no se puede culpar a ningún individuo por lo que ha ocurrido, quiere decir que el problema está en el sistema económico político" (Stiglitz, 2012, p. 32).

Sostengo que pensar alternativas a esta barbarie, no es otra cosa que pensar en (y desde) la justicia. A su vez, pensar en justicia social, inevitablemente es pensar la igualdad.

\section{Igualdad de qué}

\section{La irrupción de Rawls}

En 1971, en la universidad de Harvard, John Rawls publicó la obra que revitalizó la discusión en teoría política y en filosofía 
moral y política. Desde la tradición liberal, Rawls, se propone formular una teoría de la justicia con argumentos racionales y razonables, más allá de las posiciones utilitaristas e intuicionistas.

Uno de los tantos aspectos relevantes de Teoría de la Justicia, es que define lo que debemos entender por justicia social. Para el autor, justicia social es igualdad.

Con Rawls, y en torno a las preguntas, ¿qué es la igualdad? o ¿igualdad de qué?, se erigirá una nueva veta de la tradición liberal, el liberalismo igualitario. Integran sus filas, entre otros: Brian Barry, Ronald Dworkin o Amartia Sen, además de John Rawls, claro. Todos se posicionan en contra de la tradición libertariana, es decir, en contra del neoliberalismo y su depredación salvaje, es decir, en contra de los postulados de L. Von Mises, F. A. Hayek, o Milton Friedman, y en contra de las políticas de R. Reagan o M. Thatcher.

Tal y como sostuvimos, la piedra angular de la tradición del liberalismo igualitario es Teoría de la Justicia de John Rawls. En esa obra, Rawls, "le otorga prioridad absoluta a la Justicia y la entiende como la primera virtud de las instituciones sociales, por lo mismo en su planteamiento no le concede un valor mayor a las instituciones por presentarse ordenas, estables o eficientes, lo fundamental -menciona- será que ellas sean justas y en el caso de no serlo deberán atenerse a ser reformadas o invalidadas" (Vidal, 2009, p. 228).

Para Rawls, es básico que tanto las cargas como los beneficios sociales sean redistribuidos. Respecto a la pregunta sobre ¿igualdad de qué?, la respuesta rawlsiana apuntaría a igualar los lo que él denomina bienes primarios.

Rawls, formula dos principios que apuntan a la igualdad: a) El principio de libertad: que consiste en la igual distribución de una lista bien conocida de libertades civiles y políticas compatible con un sistema de iguales libertades para todos; y b) El principio de diferencia: de acuerdo con el cual las desigualdades sociales y económicas sólo son justificables cuando redundan en beneficio de los sectores más desposeídos de la sociedad. 
El proyecto de rawlsiano no tiene como objetivo una teoría abstracta de la justicia, por el contrario, materializa en medidas concretas los principios que se exponen como meras abstracciones. Así, para realizar el primer principio (que es premisa del segundo) señala que a cada ciudadano se le debe garantizar:

“a) los derechos y libertades básicas, [...];

b) la libertad de desplazamiento y la libre elección de ocupación en un marco de diversas oportunidades;

c) los poderes y las prerrogativas de los puestos y cargos de responsabilidad en las instituciones políticas y económicas de la estructura básica;

d) ingresos y riqueza, y por último,

e) las bases sociales de respeto a sí mismo" (Rawls, 2006, 177).

Un examen minucioso de estos principios, particularmente del principio de diferencia, permite notar que Rawls no ve en el individualismo algo propio del liberalismo. Esto, principalmente, porque rechaza la idea de mérito, es decir "la idea de que las desigualdades socioeconómicas son legítimas si estas son el resultado de diferencias debidas al talento y el esfuerzo" (Callinicos, 2006, p. 266). Para Rawls no se condice con la justicia, ni tampoco hay argumentos suficientes, para avalar el hecho de que alguien se beneficie, en desmedro de otros, de un talento o posición de la que no es merecedor realmente. En palabras de Rawls, "ninguno de los preceptos de la Justicia aspira a una virtud compensadora. Los premios obtenidos por los escasos talentos naturales, por ejemplo, han de cubrir los costes de enseñanza y alentar los esfuerzos en el aprendizaje, además de dirigir las distintas capacidades hacia donde mejor se favorezca el interés común. Las porciones distributivas resultantes no se relacionan con el valor moral, ya que la dotación inicial de activos naturales, y las contingencias de su crecimiento y educación, en las primeras etapas de la vida, son arbitrarias desde un punto de vista moral" (Rawls, 2002, p. 288). 
El principio de diferencia rawlsiano, obliga a que los mejor dotados de talentos sólo puedan beneficiarse de ellos, si aquello permite que mejore la posición de los más desfavorecidos, esto porque para Rawls el talento no es merecido, por lo que pasa a ser un activo social. Como veremos en un próximo apartado, es muy interesante que esta idea (en cierto modo), también fue formulada por Marx.

Como sostuvimos, Rawls sólo inaugura una a tradición nutrida en discusión. La pregunta de fondo en el debate es, como hemos adelantado, iigualdad de qué? De hecho, tal interrogante fue literalmente planteada por el filósofo Indio, Amartya Sen, en 1979. Cuestionarse y reflexionar en torno al contenido y alcance de la igualdad como valor, como principio o como derecho, no es, en absoluto, un asunto menor, ya que el debate abre la puerta a la radicalización de la idea de Igualdad.

\section{El debate igualitarista}

Es este debate igualitarista, el que comprueba el hecho de que la contribución rawlsiana revitaliza la discusión en filosofía práctica. Los igualitaristas buscan entrar en detalle sobre las propuestas de Rawls, criticar ciertos puntos y completar otros.

Respecto de la interrogante antes formulada, iigualdad de qué?, una respuesta posible, muy común por lo demás, es que todos deberíamos tener, al menos, la posibilidad de satisfacer nuestras necesidades de la misma forma. En esa línea argumentativa Ronald Dworkin afirma que la igualdad (correspondiente con la justicia) debe ser el remedio de lo que él llama mala suerte bruta, es decir, situaciones contingentes e involuntarias que nos ponen en desventaja respecto de los demás. Así, el principio de diferencia rawlsiano solo debería hacerse cargo de la mala suerte bruta y no de las elecciones irresponsables o suerte de opción.

Evidentemente, lo que Dworkin tiene en mente es la igualdad de recursos. Para él, todos los ciudadanos deberíamos contar con la misma cantidad de recursos y cada individuo 
deber ser libre y responsable de disponer de ellos. El hecho de usar los recursos de manera diligente, malgastarlos o despilfarrarlos dependerá de cada uno.

Dentro del debate igualitario, la posición de Dworkin tiene evidentes problemas. En primer lugar, es una concepción netamente individualista y tiene como marco una categoría propia de la derecha antiigualitaria: la elección responsable. Para Dworkin el individuo es libre de elegir y además es responsable de aquella elección casi naturalmente, cuestión con la que Milton Friedman estaría muy de acuerdo. Además, la formulación de Dworkin, adolece de un problema filosóficamente más profundo. Alex Callinicos, sostiene que Dworkin necesita que los individuos puedan distinguir entre elección (una situación subjetiva) y azar (una situación objetiva), "[p]ero las elecciones individuales y las circunstancias objetivas no son siempre tan fáciles de separar. Una persona pobre y oprimida puede reaccionar ante su situación aceptándola como parte de su destino. Puede incluso parecer que sus elecciones y preferencias reflejan satisfacción con las condiciones en las que vive. Sin embargo, alguien puede también argumentar que este es un caso en que, frente a la aparente ausencia de alternativas genuinas, las preferencias personales o individuales se han adaptado completamente a las circunstancias. Entonces, decir que la víctima de esta situación ha efectivamente elegido esta forma de vida equivaldría directamente a consagrar la injusticia. (Callinicos, 2006, pp. 268-269)"

Una tercera posición, es formulada por Amartya Sen, quien apuesta por la igualdad de capacidades. "Según Sen, aquello que deberíamos tratar de igualar es la capacidad de obtener la más amplia variedad de funcionamientos posibles. Esta postura tiene como ventaja el hecho de que nos ofrece un criterio de evaluación del bienestar (well being) individual más complejo y sutil que las crudas estadísticas del ingreso nacional difundidas por el pensamiento económico convencional" (Callinicos, 2006, p. 268). Para Sen, "[s]er libre es ser capaz, y para eso hace falta estar habilitado. Ahora bien, ¿ser capaz de qué? La respuesta es 
ser capaz de funcionar. Los funcionamientos (functionings) son las cosas que el sujeto hace o la situación en que se encuentra gracias a sus habilitaciones y al uso que pueda hacer de ellas, por ejemplo viajar, estar sano, o tener una vivienda. Los funcionamientos ofrecen un panorama de cómo es la vida del sujeto, lo cual es necesario [...] para juzgar su bienestar. Son una noción primaria en el enfoque de las capacidades, ya que éstas se formulan mediante los funcionamientos, son ellos el tipo de información más elemental que se maneja en la teoría, y son los funcionamientos, no las capacidades, lo que puede constatarse directamente" (Cejudo, 2007, p. 13).

Sen, en sintonía con marxistas como Rosa Luxemburgo o Ettiene Balibar, es muy lúcido al notar que la libertad no es concebible sin la igualdad, e incluso señala que entenderlas como conceptos distintos es un error categorial.

Sen no se muestra del todo de acuerdo con que lo que debe igualarse son los denominados bienes primarios rawlsianos, ya que las personas, por un sinnúmero de situaciones (materiales y contingentes), nunca obtendrán el mismo beneficio del conjunto de bienes primarios rawlsiano. En palabras de Sen, "[e]l juzgar los niveles de ventaja exclusivamente en función de los bienes primarios nos conduce a una moralidad parcialmente ciega" (Sen, 1994, p. 150). Las capacidades o funciones que deben igualarse son: nutrición adecuada, salud, vivienda y educación básica, entre otras cosas, lo más importante es que la igualación no debe ser cuantitativa sino que cualitativa. En tal sentido, los derechos y libertades que se garantizan a los ciudadanos deben tener una materialización real, con el objetivo de que, una vez igualadas las capacidades (como conjunto capacidades), los individuos puedan materializar realmente la libertad de elegir la forma de bienestar que ellos quieran, sólo en este momento puede operar un principio como el de diferencia.

Para discutir con Sen y con el resto de la tradición, aparece en el mapa igualitarista, Gerald Cohen. Cohen no es precisamente un liberal sino que un socialista, no en los términos del socialismo marxista (aunque sí lo fue en algún momento), sino 
que más cercano al socialismo francés e inglés anterior a Marx. Su irrupción permite sacar la discusión de los límites del liberalismo, para acercarla a las políticas de izquierda en términos amplios.

Cohen, contra Sen, repara entre otras cosas que "describir el hecho de ser saludable como una libertad o una capacidad, [es una tergiversación], puesto que se trata simplemente de una condición o un estado de existencia" (Callinicos, 2006, p. 269). Así, Cohen aboga por otra idea de igualdad, muy parecida a la de Sen, pero a su juicio más neutral. El canadiense propone la igualdad de acceso a las ventajas. "En esa propuesta, la ventaja es, al igual que el "funcionamiento" de[l] que habla Sen en su formulación más amplia, una colección heterogénea de estados deseables de la persona, que no se pueden reducir a paquetes de recursos ni a su nivel de bienes. Y mientras que "acceso" incluye lo que el término normalmente cubre, amplí[a] su significado con la estipulación de que cualquier cosa que una persona realmente tenga cuenta como algo a lo que ella tiene acceso, sin importar cómo lo ha obtenido y, por consiguiente, incluso si obtenerlo no ha implicado ninguna explotación del acceso en el sentido ordinario (ni, por lo tanto, algún ejercicio de capacidad)" (Cohen, 2004, p. 61). El proyecto de Cohen es lograr la realización de la libertad humana, una libertad que no puede entenderse, sin la igualdad, premisa marcada a fuego en la tradición socialista. Según Cohen, las desigualdades que están justificadas son aquellas que se producen por la real elección de los individuos después de igualado el acceso a las ventajas.

Las propuestas de Sen y Cohen (en cierto modo, también la de Rawls), si las llevamos a sus últimas consecuencias, afectan gravemente al sistema capitalista, incluso parecen ser incompatibles con éste. Paradójicamente, ninguno de ellos es partidario de erradicar el mercado, e incluso lo ven como la única alternativa para asignar bienes, al punto de que Cohen proponga un socialismo con mercado.

En las próximas líneas me interesa esbozar algunos contrapuntos que desde la tradición del materialismo histórico pueden hacerse a estas propuestas. 


\section{Contrapuntos a la tradición igualitarista desde el materialismo histórico}

\section{1. ¿Confianza en el mercado?}

Mercado capitalista no es sinónimo de intercambio. El sistema y el mercado capitalista, son creaciones propiamente modernas. El mercado no es natural, a pesar de lo que podría creerse. En el mercado existe intercambio, pero no necesariamente todo intercambio se hace en el marco del mercado capitalista. Mientras que el mercado necesariamente se rige por los beneficios que se obtengan (del intercambio), el intercambio sólo tiene por objeto la transferencia de una o más cosas. En definitiva, criticar el mercado capitalista, en ningún sentido es equivalente a oponerse al intercambio de bienes (cuestión absolutamente necesaria en la vida en sociedad), perfectamente puede existir intercambio sin mercado capitalista.

¿Cuál es el problema con el mercado? Sobre esto podemos argumentar desde dos posiciones, más complementarias que antagónicas.

Ellen Meiksins Wood nos llama a recuperar la democracia en todo ámbito de la vida humana. Para ella, la democracia no es una mera forma de organizar lo político, entendiendo por esto algo separado de lo económico. Entender que la economía puede abstraerse del resto de las relaciones sociales es un sinsentido. Para Meiksins Wood la separación entre lo económico y lo extraeconómico no es del todo correcto ya que lo económico permea lo extraeconómico y viceversa. Es por eso, que el mercado no puede eximirse de un análisis y crítica igualitaria (y democrática) si se quiere ser riguroso.

El capitalismo y el mercado capitalista son por esencia antiigualitarios y antidemocráticos. En palabras de Meiksins Wood, "el capitalismo es estructuralmente antitético respecto de la democracia, en principio, por la razón histórica más obvia: no ha existido nunca una sociedad capitalista en la cual no se le haya asignado a la riqueza un acceso privilegiado al poder" (Meiksins 
Wood, 2006, p. 396). En el mercado capitalista las decisiones y el poder están estrechamente ligadas a la acumulación, a la capacidad de obtener ganancias y a la cantidad de las mismas. Las políticas igualitarias no pueden estar arrojadas a una institución que opera en la antítesis de la igualdad. Un proyecto realmente emancipador e igualitario debe "tener diversidad, diferencia y pluralismo; pero no un pluralismo carente de estructura e indiferenciado. [...] Esto significa un pluralismo que reconozca la unidad sistémica del capitalismo y pueda distinguir las relaciones constitutivas del capitalismo de otras desigualdades y opresiones. [...] No debemos confundir respeto con pluralidad de la experiencia humana, ni luchas sociales con una completa disolución de la causalidad histórica" (Meiksins Wood, 2000, p. 305).

Otra posición, pero en la misma línea es la del chileno Carlos Pérez Soto, quien aboga por ampliar la noción de valor y de explotación. Desde esta perspectiva, "[h]ay explotación cuando hay intercambio desigual de valor. Hay que recordar, por supuesto, que [para Pérez] el valor es inconmensurable. Esto significa que el juicio "explotación" refiere no al intercambio como tal, sino a la ficción de equivalencia que lo preside. Hay explotación cuando, dada una Ficción de equivalencia, aun en sus propios términos, el intercambio resulta desigual" (Pérez Soto, 2008, p. 113). Así, la desvalorización del hombre por el hombre, puede ser tachada de injusta.

Con el objetivo de comprender más cabalmente esta crítica, es necesario ahondar en el concepto de explotación.

\section{Igualdad y explotación capitalista}

La tradición del materialismo histórico, entre las tantas críticas que ha formulado a la formación social capitalista, ha evidenciado la explotación capitalista como forma de obtener ganancia. A su vez, a toda la tradición igualitarista, podemos criticar que omiten el problema de la explotación capitalista.

El capitalismo es aquella formación social en el que la producción es organizada y dominada por un sujeto colectivo 
al que le pertenecen los medios de producción; por otro lado, existe otro sujeto colectivo que no posee medios de producción, sólo posee su fuerza de trabajo. Por esa razón, debe someterse y producir con los medios de producción que pertenecen al primer grupo, bajo las órdenes de éste. Estos sujetos colectivos son las clases sociales.

Lo determinante es que la clase dominante controla la división social del trabajo, mientras que la clase dominada no. La clase dominada se somete a la clase dominante, somete su cuerpo y su tiempo.

Es interesante recordar que para los marxistas, en concordancia con lo que ya habían señalado liberales como Adam Smith o David Ricardo, lo que valoriza un objeto es el trabajo humano, es decir, es el trabajador a través de su trabajo el que permite que el objeto producido tenga un determinado valor, más allá del valor que ya tengan las materias primas con las que se trabaje. En los términos de Marx, lo que determina la magnitud del valor de una mercancía "es la cantidad de trabajo socialmente necesario o el tiempo socialmente necesario [para producirla]" (Marx, 2010, p. 53).

Por el trabajo realizado, el trabajador recibe un salario. Esta es una típica relación de intercambio. Lo particular de esta relación de intercambio es que se hace dentro del mercado (del trabajo) y es considerada una relación de intercambio equivalente.

Lo que nos interesa destacar de todo esto, es que dentro del capitalismo todo intercambio que se considere equivalente, está fundado en una ficción, "una ficción de equivalencia, acordada o impuesta" (Pérez Soto, 2008, p. 100). En el mercado capitalista, toda la producción humana se puede poner en venta como mercancía.

El problema surge cuando el intercambio se considera equivalente pero materialmente es desigual. A este problema le llamamos explotación. Existe explotación cuando, operando una ficción de intercambio equivalente, aun en sus propios términos, el intercambio resulta desigual. 
Ocurre que la igualdad entre trabajador y capitalista es meramente formal, debido a que el trabajador si bien es libre políticamente y legalmente, y además es libre de disponer de su fuerza de trabajo como su propia mercancía, al mismo tiempo, de la única mercancía real de la que dispone es de su propia fuerza de trabajo. "Como se le ha denegado el acceso a los medios de producción, está obligado por las condiciones sociales a vender la totalidad de su vida activa, su capacidad para el trabajo. El capitalista utiliza su control de los medios de producción para concertar una negociación sumamente ventajosa: una vez empleado, el trabajador produce mercancías para el capitalista bajo el control de este último a cambio de un salario que representa tan sólo una parte del valor [o del capital] que crea. La aparente libertad e igualdad del trabajador respecto al capitalista oculta una subordinación y desigualdad subyacentes cuyo resultado es la explotación del primero" (Callinicos, 2003 (a), p. 41). En definitiva, la explotación consiste en la apropiación que la clase dominante hace del valor producido por la clase dominada y que no le es devuelto a través del salario.

Entonces, ¿cuál es el problema? El problema del capitalismo y del mercado, es que las relaciones de producción (ya no de objetos, sino de la vida en general) cosifican a los sujetos según su rol histórico. En este sentido, la humanidad queda dividida, no en términos monetarios sino respecto del rol que juegan en la división social del trabajo. "En efecto, a partir del momento en que comienza a dividirse el trabajo, cada cual se mueve en un determinado círculo exclusivo de actividades, que le es impuesto y del que no puede salirse; el hombre es cazador, pescador, pastor o crítico, y no tiene más remedio que seguirlo siendo, si no quiere verse privado de los medios de vida" (Marx, 1986, p. 36). Es paradójico que en el capitalismo toda producción es social, pero la acumulación de las ganancias es siempre individual.

No considerar la explotación, es omitir de manera grosera un problema claro. Esta omisión es una debilidad fuerte de la tradición igualitaria. La explotación capitalista permite a una 
clase apropiarse de los frutos del trabajo de la otra, es decir, es permitir que el bienestar material de unos dependa del sacrificio de otros.

Las instituciones que hace permisible la explotación capitalista son la igualdad formal ante la ley y el contrato de trabajo. El mismo Marx, en El Capital, señala sobre dichas instituciones que "[1]a órbita de la circulación o del intercambio de mercancías, en cuyo marco se desenvuelve la compra y venta de la fuerza de trabajo, era, en realidad, un verdadero edén de los derechos innatos del hombre. Dentro de sus límites imperan la libertad, la Igualdad, la propiedad y Bentham. ¡La libertad! pues el comprador y el vendedor de una mercancía, por ejemplo la fuerza de trabajo, se someten sólo a su libre voluntad. Contratan como hombres libres e iguales jurídicamente. El contrato es el resultado final en que sus voluntades cobran una expresión jurídica común. ¡La Igualdad!, pues compradores y vendedores se refieren recíprocamente sólo como poseedores de mercancías, cambiando equivalente por equivalente. ¡La propiedad!, pues cada uno dispone únicamente de lo que posee. ¡Y Bentham!, pues cada uno se preocupa únicamente de sí mismo" (Marx, 2010, pp. 182-183). Es cierto que en términos de intercambio capitalista, detrás del contrato de trabajo hay una ficción de equivalencia, es decir, las prestaciones se miran como iguales. Pero ocurre que esta ficción de equivalencia oculta una relación que Marx llamó muchas veces llamó robo, pero que nunca tachó como injusta, pero considerando los hechos de manera real es evidentemente injusta.

En términos estrictos, explotadores y explotados no son libres de la misma manera (y por lo mismo, nunca serán iguales), la supervivencia de los trabajadores depende del salario que se les pague, que no es más que una parte del valor que producen. Su igualdad es aparente ya que su relación real es de subordinación y dependencia, de fondo lo que existe es explotación.

En ese sentido, la explotación constituye dos sujetos en situaciones desiguales que no son elegidas y que se originan 
con la institución misma. De esta forma, el problema central de la explotación no es la forma en que se distribuye el producto social, es decir, no se soluciona con la redistribución de la riqueza sino que terminando con lo que origina las relaciones de explotación, en otras palabras, terminando con el capitalismo.

Desde un punto de vista ético, se puede argumentar que la apropiación y explotación capitalista son injustas. El problema de argumentar desde esta perspectiva es que hay un contraargumento que genera equivalencia. Quienes están a favor del capitalismo pueden argumentar (y lo hacen) que la apropiación puede ser privada debido a que los dueños del capital y los medios de producción se arriesgan y compiten en el mercado capitalista. Así, tal y como argumenta Hayek, los resultados del mercado no pueden considerarse justos ni injustos. En tal sentido hay equivalencia, equivalencia argumentativa que es fiel reflejo de la lucha de clases, en la cual un sujeto histórico cree tener derecho al valor apropiado (la clase explotadora), mientras que el otro cree tener derecho a socializar ese valor (la clase explotada).

Alex Callinicos se ha preocupado de mostrar que detrás de esta equivalencia argumentativa hay algunas apariencias, que develándolas permiten argumentar que el capitalismo es injusto. Callinicos señala que "[b]ajo el capitalismo, los individuos no gozan del mismo acceso a las ventajas. No sólo son tremendamente desiguales el acceso a los recursos de producción y la distribución de la riqueza y los ingresos, sino que las oportunidades que se presentan a los individuos durante su vida se ven enormemente afectadas, para bien o para mal, por procesos que caen fuera de su control, en particular las fluctuaciones del mercado. Basta con pensar en las fortunas creadas, pero también en las vidas destruidas, por los auges y debacles de la era neoliberal. No es de extrañar que Friedrich von Hayek, probablemente el más sofisticado defensor del capitalismo, se opusiera con vehemencia a apelar a ninguna concepción de Justicia social al evaluar los méritos relativos de los sistemas económicos" (Callinicos, 2003 (b), pp. 141-142). 
Por otro lado, Ellen Meiksins Wood presenta otro argumento, siguiendo la misma línea, que nos parece demoledor. "Capitalismo y democracia son incompatibles también, y principalmente, porque la existencia del capitalismo depende de la sujeción a los dictados de la acumulación capitalista y las "leyes" del mercado de las condiciones básicas de vida y reproducción social como condición irreductible contraria al ánimo democrático. Esto significa que el capitalismo necesariamente sitúa cada vez más esferas de la vida cotidiana por fuera del parámetro según el cual la democracia debe rendir cuentas de sus actos y asumir responsabilidades. Toda práctica humana que pueda ser convertida en mercancía deja de ser accesible al poder democrático. Esto quiere decir que la democratización debe ir de la mano de la "desmercantilización". Pero desmercantilización significa, por definición, el final del capitalismo" (Meiksins Wood, 2006, p. 396). En este sentido, la mercantilización de todo es totalmente incompatible con la verdadera democracia, aquella en que somos dueños no sólo de nosotros mismos, sino capaces de decidir, no sólo políticamente sino que en todo ámbito de cosas.

Llegados a este punto, me parece que hay razones suficientes como para sostener que la idea de igualdad por la que debemos trabajar no debe ser humanizar el capitalismo, sino que erradicarlo.

\section{Marx en el debate igualitario}

La igualdad es un valor que quienes buscamos contribuir a la tradición del materialismo histórico (ya sea desde el marxismo o desde el anarquismo) debemos rescatar. No sólo debemos señalar que la justicia está de nuestro lado o señalar que nuestras demandas son justas en un sentido meramente retórico. Es cierto que a Marx el tema de la justicia no le importó de mayor manera, o al menos no lo desarrolló, pero para la tradición materialista e historicista, hoy es imperativo pensarla. Es por eso que nos parece que la discusión que los igualitaristas dieron 
dentro de su tradición no debe ser ignorada o vapuleada sin mayores fundamentos.

El debate igualitarista, y los textos de Marx y la tradición marxista, desde nuestro punto de vista, sirven de insumos para construir políticas igualitarias desde el materialismo histórico.

Si nuestro horizonte es una sociedad sin clases, una sociedad libre e igualitaria, necesitamos pensar la justicia. "Según Marx, el comunismo no es la forma final de la sociedad humana; es el comienzo de la historia auténticamente humana" (Lizarraga, 2011, p. 78).

Una parte no menor de la izquierda podría argumentar que la justicia no importa, que la sociedad comunista sobrepasa la justicia. Contra aquello, el argentino, Fernando Lizarraga señala que: "habrá quienes sostengan que el cambio revolucionario es un evento puro que funda su propia legitimidad y no precisa invocar principios anteriores a ella; pero esto se da de bruces con la concepción del marxismo como filosofía de la praxis y de la revolución como un cambio material, intelectual y moral. Habrá aún quienes sostengan que el comunismo es una sociedad más allá de la Justicia, porque la abundancia será tal que las cuestiones distributivas nunca habrán de plantearse; pero esto ignora los evidentes límites en los recursos planetarios y las opciones éticas que plantea la noción de límite" (Lizarraga, 2008, p. 85).

Es cierto que los niveles de sobreproducción capitalista alcanzan para que sean distribuidos por toda la población mundial, pero no es menos cierto que el costo a nivel ecológico es altísimo. Si queremos otro mundo donde sea posible realizar la historia realmente humana, es necesario que las condiciones de producción sean humanizadas, además de que consideren y conserven el mundo natural que las hace posibles.

El proyecto al que apuntamos, es un proyecto crítico. Prescinde del mercado y busca superar el capitalismo por todas las razones que ya hemos especificado, Abogamos por un sistema donde "cada individuo no [tenga] acotado un círculo exclusivo 
de actividades, sino que puede desarrollar sus aptitudes en la rama que mejor le parezca" (Lizarraga, 2011, p. 78), esto sólo es posible si la sociedad (en general) se encarga de regular y planificar la producción general.

En la tradición histórico-materialista, criticar es superar. Es decir, criticar el capitalismo implica pensar, a la vez, su superación. En tal sentido, criticar el capitalismo implica, a lo menos, esbozar un proyecto distinto.

Sostenemos que es posible construir un marco ético normativo para una sociedad más allá del capitalismo. Este marco obvia y necesariamente, defiende una ética liberadora, una ética de la emancipación.

Para su construcción, se consideran los principios implícitos en la Crítica del programa de Gotha, a saber, el principio de contribución y el principio de necesidad, además del principio de diferencia rawlsiano. Estos principios tienen en su horizonte la creación de una "asociación en que el libre desenvolvimiento de cada uno se[a] la condición del libre desenvolvimiento de todos" (Marx y Engels, 2000, p. 66)

\section{El Principio de Contribución}

En la ya referida Crítica al programa de Gotha, Marx, esboza el principio que debería regir a la primera fase de la sociedad comunista, fase a la que Lenin llamó socialismo.

El principio de contribución responde a una concepción del comunismo y de la revolución como procesos dinámicos. Es claro que para que este principio pueda actuar debe cumplirse con ciertos aspectos mínimos que, en definitiva, hacen posible su aplicación.

La aplicación del principio de contribución se funda necesariamente en la socialización de los medios de producción y en el control colectivo de los mismos, es decir, la clase trabajadora debe ser quien organice tanto la producción como la distribución. 
Marx estaba lejos de ser un iluso, y tenía muy claro que aún dándose estos supuestos, la primera fase de la sociedad comunista estaría mancillada por elementos en los que se funda la superada sociedad de clases. Es por esta razón que la primera fase de la sociedad comunista responde al principio de contribución.

Muy a grandes rasgos, este principio se explica en una simple frase: a cada quien según su trabajo pero, claramente, el asunto es más complejo que lo esbozado. En palabra de Marx el principio puede explicarse de la siguiente forma: "De lo que aquí se trata no es de una sociedad comunista que se ha desarrollado sobre su propia base, sino de una que acaba de salir precisamente de la sociedad capitalista y que, por tanto, presenta todavía en todos sus aspectos, en el económico, en el moral y en el intelectual, el sello de la vieja sociedad de cuya entraña procede. Congruentemente con esto, en ella el productor individual obtiene de la sociedad -después de hechas las obligadas deducciones- exactamente lo que ha dado. Lo que el productor ha dado a la sociedad es su cuota individual de trabajo. Así, por ejemplo, la jornada social de trabajo se compone de la suma de las horas de trabajo individual; el tiempo individual de trabajo de cada productor por separado es la parte de la jornada social de trabajo que él aporta, su participación en ella. La sociedad le entrega un bono consignando que ha rendido tal o cual cantidad de trabajo (después de descontar lo que ha trabajado para el fondo común), y con este bono saca de los depósitos sociales de medios de consumo la parte equivalente a la cantidad de trabajo que rindió" (Marx, 2000, p. 15).

De la explicación hecha por el mismo Marx, puede extraerse que todo trabajador recibe una cuota proporcional a su trabajo después de que se han hecho los descuentos necesarios, o más claro aún "de cada quien según su capacidad a cada quien proporcionalmente a su contribución laboral, después de que se han separado fondos para satisfacer necesidades económicas y necesidades comunes" (Lizarraga, 2011, p. 99). Es necesario aclarar un punto que está implícito en la formulación de este 
principio, y es que todos quienes estén habilitados para trabajar deben hacerlo, quienes están imposibilitados de hacerlo serán asistidos mediante fondos de necesidades comunes.

En definitiva, el principio de contribución es un principio igualitario desde un punto de vista bastante simple, y es que permite el acceso igualitario a los medios de producción, pero al estar influenciado por el derecho burgués es, por otro lado, un principio no-igualitario, ya que no considera las desigualdades producto de las condiciones físicas o intelectuales, es decir no se hace cargo de la diversidad de los trabajadores. Los resultados a los que conlleva el principio de contribución pueden satisfacer a un trabajador con muy buenas condiciones o talentos pero puede no satisfacer a un trabajador al que sus condiciones físicas o intelectuales no le sean suficientes, por esta razón sostengo que el principio de contribución debe ser complementado por el principio de diferencia rawlsiano. Esto permite dar un enfoque aún más igualitario a la primera fase del comunismo, ya que permite corregir las desigualdades derivadas del primer principio pensado por Marx

\section{El principio de Diferencia}

Discutir con la tradición igualitaria puede traer algunos réditos. En este caso en particular, permite añadir un criterio aún más igualitario al principio de contribución.

El principio de diferencia es aquel que señala que "las desigualdades sociales y económicas habrán de ser conformadas de modo tal que: a) se espere razonablemente que sean ventajosas para todos, b) se vinculen a empleos y cargos asequibles para todos" (Rawls, 2002, p. 266), en otras palabras, este principio atiende a que las desigualdades sociales y económicas sólo son justificables cuando redundan en beneficio de los sectores más desposeídos de la sociedad, esto debe sumarse a la igualdad de oportunidades.

Anteriormente, sostuvimos que el principio de contribución no es del todo igualitario, ya que no considera a los trabajadores 
en particular, sino que en general. Tal como el derecho burgués, es un principio de derecho igual que no se hace cargo de sus consecuencias desiguales. De esta forma, el principio de necesidad permite amortiguar las consecuencias derivadas de factores contingentes y moralmente arbitrarios como la lotería física o de talentos.

Con el principio de contribución, los más aventajados en dicha lotería podrían sacar provecho considerable de algo que en realidad no merecen (ya que por ejemplo no pone límites a la cantidad de ingresos que podrían recibir) y los menos aventajados deberían sufrir por algo de lo que no son responsables, pero al agregar el principio de diferencia al principio de contribución, las consecuencias no igualitarias del primer principio pueden ser aminoradas.

Así, los más aventajados en talentos o cualidades físicas sólo podrán beneficiarse de las desigualdades que beneficien a la vez a los desaventajados. De esta forma, sólo son tolerables desigualdades que beneficien a todos.

Hasta aquí, damos por reproducidos todos los supuestos y elementos que esbozamos para el principio de contribución pero agregamos que sólo serán tolerables las desigualdades que vengan en el beneficio de todos. De esta forma, podríamos formular el nuevo principio de contribución de este modo: de cada quien según su capacidad a cada quien proporcionalmente a su contribución laboral, después de que se han separado fondos para satisfacer necesidades económicas y necesidades comunes; aceptando sólo las desigualdades que vengan en beneficio de todos

Creemos que en este punto, tal y como adelantamos, Rawls y Marx se encuentran en total sintonía. Si bien Marx no formuló el principio de diferencia, sí hizo referencia implícita pero clara a este principio. En su Crítica al programa de Gotha, Marx se muestra en contrario a los privilegios naturales y al derecho igual. "Por lo visto, cabe sospechar que Marx tenía el principio de diferencia en la punta de la lengua, porque identificaba los talentos naturales y circunstancias sociales como factores que 
deben ser neutralizados al escoger principios de justicia" (Lizarraga, 2011, p. 104). De esta forma, tanto Rawls como Marx convergen en su rechazo a la autopropiedad formulada por Nozick, toda vez que los talentos o privilegios naturales son arrojados a un acervo común y no son aprovechados individualmente. En Marx, la razón es simple, si la producción es social, el aprovechamiento de los beneficios debe serlo también.

\section{El Principio de Necesidad}

Para el momento que se haya superado todo residuo de la antigua sociedad burguesa, Marx pensó un segundo principio distributivo, denominado principio de necesidad. Este principio es quizás una de las máximas más conocidas respecto de las formulaciones de Marx. En palabras de Marx, el asunto es como sigue, "En la fase superior de la sociedad comunista, cuando haya desaparecido la subordinación esclavizadora de los individuos a la división del trabajo, y con ella, la oposición entre el trabajo intelectual y el trabajo manual; cuando el trabajo no sea solamente un medio de vida, sino la primera necesidad vital; cuando, con el desarrollo de los individuos en todos sus aspectos, crezcan también las fuerzas productivas y corran a chorro lleno los manantiales de la riqueza colectiva, sólo entonces podrá rebasarse totalmente el estrecho horizonte del derecho burgués, y la sociedad podrá escribir en su bandera: ¡De cada cual, según sus capacidades; a cada cual, según sus necesidades!" (Marx, 2000, p. 17).

Si se conoce la ampliamente apoyada tesis marxista de que la fase superior del comunismo supera o excede la justicia, carece de sentido sostener que el principio de necesidad es un principio de justicia. Es por esta razón que es totalmente legítimo preguntarse, en qué sentido el principio de necesidad es un principio igualitario. Si bien es cierto que Marx se no está del todo conforme cuando utiliza conceptos ético-normativos, claramente, no rechaza la idea de igualdad, pero desde criterios distintos, como ya lo hemos venido sosteniendo. 
Hoy este asunto toma especial relevancia, debido a que los daños ecológicos que el capitalismo ha hecho a nuestro planeta parecen irreversibles, de modo que la superabundancia que Marx y gran parte de la tradición marxista imaginaron, hoy carece de sentido. El comunismo es posible en la abundancia, claro está, pero no es necesario sobreproducir de la forma en que hoy se hace. Es por esta razón que sostenemos que los tres principios esbozados son principios de justicia, ya que permiten pensar la distribución de los bienes desde la igualdad.

Volviendo al principio de necesidad y a la pregunta formulada, el principio de necesidad es un principio de justicia toda vez implica un "igualitarismo complejo" (Lizarraga, 2011, p. 236). Aquí hay un criterio moral común a igualar, las necesidades, pero la materialización de este principio no considera resultados cuantitativos sino que cualitativos. A este principio no le interesa cuanto se tenga sino que ce centra en la calidad de lo que se tenga.

Respecto del concepto de necesidades cabe destacar lo siguiente. En la Ideología Alemana, Marx señala que "el primer hecho histórico es, por consiguiente, la producción de los medios indispensables para la satisfacción de estas necesidades, es decir, la producción de la vida material misma [...] Lo segundo es que la satisfacción de esta primera necesitad, la acción de satisfacerla y la adquisición del instrumento necesario para ello conduce a nuevas necesidades y esa creación de necesidades nuevas constituye el primer hecho histórico" (Marx, 1986, p. 28). Con esto, queremos decir que se produce debido a que existen necesidades, pero lo que se produce al satisfacer necesidades, son necesidades. De esta forma, las necesidades son producidas, no naturales. Marx utiliza un razonamiento circular para explicar que lo que se produce en la producción, es el hombre mismo y las necesidades de éste son sociales y no naturales como podría pensarse. La esencia del hombre es que produce su propia vida. De esta forma las necesidades entendidas de esta manera, permiten formular un criterio igualitario. Para sostener esto, solo tenemos que explicar una idea básica, 
sostenemos que justicia es igualdad, y la igualdad atiende a que todos y todas tengamos el mismo acceso a los recursos que necesitamos para vivir la vida que valoramos. De esta forma, las necesidades no deben entenderse como bienes, sino como capacidades que permitan, en los términos de Agnes Heller, el bienestar, la proyectividad y la afectividad.

Tal y como puede observarse, al presentar el proyecto de esta forma, es innegable que dentro del proyecto del mismo Marx, la igualdad es central, pero su noción de igualdad es claramente una igualdad diferenciada, eso es claro y se condice con el objetivo del proyecto en el que se embarcó. El proyecto que busca terminar con la historia de la lucha de clases, para comenzar a escribir la historia humana.

\section{Consideraciones finales}

Marx no quiso pensar sociedades futuras. Sí señaló que la única salida al conflicto de clases es la revolución, pero ¿después qué? Plantearnos alternativas más allá del mercado y del capitalismo, es para la izquierda una tarea urgente, una de las principales razones es que "el vuelo a ciegas del capitalismo, impelido por los procesos de acumulación competitiva [...] ha producido una forma de desarrollo económico que es a todas luces insostenible desde un punto de vista ecológico" (Callinicos, 2003 (b), 141-142) .

Nuestro horizonte debe ir más allá del capitalismo y del mercado, pero también debe rechazar la propuesta centralista y antidemocrática soviética, reconocerla como un real desastre, es más, debemos entenderla como capitalismo de Estado, capitalismo burocrático.

"Desde luego que no se trata de diseñar sociedades de fantasía, donde cada ínfimo detalle ha sido considerado hasta la exasperación; ni tiene mucho sentido embarcarse en la elaboración de ingeniosos experimentos mentales a menos que se tome nota de los movimientos emancipatorios realmente existentes" (Callinicos, 2003 (a), p. 160). Callinicos propone recurrir 
a la imaginación utópica, "esa capacidad para anticipar, al menos como bosquejo, una forma de coordinación económica eficiente, democrática y ajena al mercado" (Callinicos, 2003 (a), p. 160). Todas las alternativas deben ser levantadas atendiendo a valores como la justicia; la democracia; la eficiencia; la solidaridad y la sustentabilidad. Proponer valores acorde con una noción anticapitalista de la igualdad es, en otras palabras, construir un ethos, pero uno que se origine y se desarrolle en la práctica política.

Desde un tiempo hasta ahora, se han levantado dos proyectos muy interesantes de no-mercado: la Coordinación negociada de Pat Devine y la economía participativa o PARECON de Michael Albert y Robbin Hannel. Estos proyectos tienen en común que la producción no está determinada por los mercados o por una clase burocrática, sino por los trabajadores y los consumidores mismos, son proyectos realmente participativos, donde "la democracia deja de ser un simple mecanismo decisorio al estilo schumpeteriano y se convierte en una genuina categoría política, social y económica" (Lizarraga, 2010, p. 91).

Podemos optar por la coordinación negociada de Devine, tal como propone Callinicos; por la PARECON de Albert, una alternativa más libertaria que la de Devine; o construir otras con los principios marxianos que ya esbozamos. Lo central es "terminar con la división social del trabajo y pasar a una división funcional de éste, así las tareas no estarían determinadas e institucionalizadas; disminuir las jornadas de trabajo, distinguir entre trabajo libre y productivo (muchos trabajos actuales serían trabajos libres) donde todos tengamos que participar de aquella distinción" (Gres, 2011, 369). Luchar por un ingreso básico universal por el solo hecho de existir, y por la socialización de los medios de producción.

Por último, los invito a pensar, imaginar y discutir proyectos posibles. Si nuestro horizonte es una sociedad sin clases debemos considerar la igualdad, como igualdad diferenciada y precisamente reconocer toda diferencia no basada en la explotación, opresión o dominación, debemos preocuparnos 
de construir proyectos políticos y económicos democráticos. Si nuestro horizonte requiere de un orden, pero no de un sistema que favorezca estructuralmente a una clase minoritaria, debemos pensar y preocuparnos de la igualdad.

\section{Referencias bibliográficas}

Albert, Michael. (2003). Economía participativa. Conferencia en Woods Hole. Estados Unidos. Woods Hole.

Callinicos, Alex. (2006) "Igualdad y Capitalismo". En: BORON, Atilio; AMADEO, Javier y GONZÁLEZ, Sabrina (comp.). La teoría marxista boy: Problemáticas y perspectivas. CLACSO. Buenos Aires.

- (2003 a). Igualdad. Madrid. Siglo XXI.

- (2003 b). Un Manifiesto anticapitalista. Barcelona. Crítica.

Cejudo, Rafael. Capacidades y Libertad: "Una aproximación a la teoría de Amartya Sen". En: Revista Internacional de Sociología (RIS). No 47. 2007, pp. 9-22.

Cohen, G.A. (2004). "iIgualdad de qué? Sobre el bienestar, los bienes y las capacidades". En: NUSSBAUM, Martha y SEN, Amartya (comp.). La Calidad de vida. México D.F. Fondo de Cultura Económica.

FAO (Organización de las Naciones Unidas para la Alimentación y la Agricultura). (2012). Nuevo informe sobre el hambre: casi 870 millones de personas sufren subnutrición crónica en el mundo. Roma. En línea.

Gres Chávez, Pablo. (2011). "Ciudadanía y Ética Empresarial: Análisis crítico y contribuciones para un proyecto de Participación Económica", en: Derecho y Humanidades. Número 19. Santiago: Universidad de Chile, pp. 349-371.

Hobsbawm, Eric. (1999). Historia del siglo XX. Crítica. Buenos Aires. Kelsen, Hans. (1957). What is Justice?. Berkeley. California.

Lizarraga, Fernando. (2011). El Marxismo y la Justicia Social. Santiago, Plataforma Nexos.

- (2008). Por un diálogo entre marxismo e igualitarismo liberal. En: Ruth. Cuadernos de pensamiento crítico. Ciudad de Panamá, pp. 68-87. 
- (2010). Utopías posibles más allá del mercado, en: Otros Logos. Revista de estudios críticos. Año 1, Número 1. Centro de Estudios y Actualización en Pensamiento Político, Decolonialidad e Interculturalidad. Neuquén, Argentina: Universidad Nacional del Comahue, pp. 70-93.

Marx, Karl. (2000). Crítica al programa de Gotha. Buenos Aires. ElAleph

- (2010). El capital: crítica de la economía política. Tomo I. Santiago. LOM.

- (1986). La ideología alemana. Barcelona. Grijalbo

Marx, Karl / Engels, Friedrich. (2000). Manifiesto del Partido Comunista. Buenos Aires. ElAleph.

Meiksins Wood, Ellen. (2006). "Estado, Democracia y Globalización", En: Boron, Atilio; Amadeo, Javier y González, Sabrina (comp.). La teoría marxista boy: Problemáticas y perspectivas. CLACSO. Buenos Aires.

Mollard, Carlos. (2012). La pobreza no está en la agenda empresarial. Sin Mordaza. Buenos Aires En línea.

OXFAM (Oxford Committee for Famine Relief). 2016. Informe $\mathrm{N}^{\circ}$ 210. OXFAM. 2016

Pérez Soto, Carlos. (2008). Proposición de un Marxismo hegeliano. Santiago. Arcis-LOM.

Rawls, John. (2006). Liberalismo Político. México D.F. Fondo de Cultura Económica.

- (2002). Teoría de la Justicia. Madrid. Fondo de Cultura Económica

Sen, Amartya. (1994). ¿Igualdad de qué?. En: VV.AA. Libertad, Igualdad y Derecho. Barcelona. Planeta De Agostini

Stiglitz, Joseph. (2012). El precio de la desigualdad. Madrid. Taurus. UNDP (United Nations Development Programme). (1999). Human Development Report.

Vidal, Paula. (2009). La teoría de la justicia social en Rawls: ¿Suficiente para enfrentar las consecuencias del capitalismo? En: Polis. Año 9, No 23. pp. 225-246. 